\title{
Prevalence of Coronary Heart Disease Risk Factors and Screening for High Cholesterol Levels Among Young Adults, United States, 1999-2006
}

\author{
Elena $V$. Kuklina, $M D, P b D$ \\ Paula W. Yoon, ScD, MPH \\ Nora L. Keenan, PbD
}

Division for Heart Disease and Stroke Prevention, National Center for Chronic Disease Prevention and Health Promotion, Centers for Disease Control and Prevention, Atlanta, Georgia
Conflicts of interest: none reported

\section{CORRESPONDING AUTHOR}

Elena V. Kuklina, MD, PhD

Division for Heart Disease

and Stroke Prevention

Centers for Disease Control and Prevention 4770 Buford Hwy, NE, Mailstop K-47

Atlanta, GA 30341

ekuklina@cdc.gov

\begin{abstract}
PURPOSE Previous studies have reported low rates of screening for high cholesterol levels among young adults in the United States. Although recommendations for screening young adults without risk factors for coronary heart disease (CHD) differ, all guidelines recommend screening adults with CHD, CHD equivalents, or 1 or more CHD risk factors. This study examined national prevalence of $\mathrm{CHD}$ risk factors and compliance with the cholesterol screening guidelines among young adults.
\end{abstract}

METHODS National estimates were obtained using results for 2,587 young adults (men aged 20 to 35 years; women aged 20 to 45 years) from the 1999-2006 National Health and Nutrition Examination Surveys. We defined high low-density lipoprotein cholesterol (LDL-C) as levels higher than the goal specific for each CHD risk category outlined in the National Cholesterol Education Program Adult Treatment Panel III guidelines.

RESULTS About $59 \%$ of young adults had CHD or CHD equivalents, or 1 or more of the following CHD risk factors: family history of early CHD, smoking, hypertension, or obesity. In our study, the overall screening rate in this population was less than $50 \%$. Moreover, no significant difference in screening rates between young adults with no risk factors and their counterparts with 1 or more risk factors was found even after adjustment for sociodemographic and health care factors. Approximately $65 \%$ of young adults with CHD or CHD equivalents, $26 \%$ of young adults with 2 or more risk factors, $12 \%$ of young adults with 1 risk factor, and $7 \%$ with no risk factor had a high level of LDL-C.

CONCLUSIONS CHD risk factors are common in young adults but do not appear to alter screening rates. Improvement of risk assessment and management for cardiovascular disease among young adults is warranted.

Ann Fam Med 2010;8:327-333. doi:10.1370/afm.1137.

\section{INTRODUCTION}

$\mathrm{n}$ abnormal lipid profile is a highly common but modifiable risk fac-
tor for coronary heart disease (CHD), a leading cause of mortality
in the United States. The importance of screening for identification and clinical management of dyslipidemia is recognized by several professional associations and public health organizations. ${ }^{1,2}$ The National Cholesterol Education Program Adult Treatment Panel III (NCEP ATP III), endorsed by the American Heart Association and the National Heart, Lung, and Blood Institute, recommends universal screening for high cholesterol levels beginning at age 20 years. ${ }^{2}$ The US Preventive Services Task Force (USPSTF) advocates a targeted cholesterol screening approach for young adults (men aged 20 to 35 years and women aged 20 to 
45 years). The USPSTF recommends screening young adults with CHD, CHD equivalents (other forms of atherosclerotic vascular disease, diabetes, or CHD risk of $20 \%$ or greater within 10 years), or 1 or more CHD risk factors (high blood pressure, smoking, family history, and obesity). ${ }^{1}$ There are no specific USPSTF recommendations for persons who do not have CHD risk factors. Thus although screening guidelines vary, there is agreement concerning the need to screen young adults who are at increased risk of CHD.

Previous reports based on the Behavioral Risk Factor Surveillance System data indicated a low screening rate among young adults. In 2003 only about $60 \%$ of US adults aged 20 to 44 years had had their cholesterol levels checked in the preceding 5 years, compared with $85 \%$ and $89 \%$ of adults aged 45 to 64 years and 65 years and older, respectively. ${ }^{3}$ Although the use of drug therapy to treat high levels of low-density lipoprotein cholesterol (LDL-C) may be indicated in a small proportion of young adults, therapeutic lifestyle changes would be sufficient to manage high LDL-C among most young adults. ${ }^{2}$ Data on compliance with cholesterol-screening guidelines among young adults and the prevalence of high LDL-C are scarce, however. This study, which relied on data for 1999 to 2006 from the National Health and Nutrition Examination Survey (NHANES) of young adults (men aged 20 to 35 years and women aged 20 to 45 years), had 3 objectives to determine: (1) the proportion of persons with CHD, CHD equivalents, or CHD risk factors $_{i}(2)$ rates of the self-reported screening for high cholesterol levels that had been performed before the study; and (3) the prevalence of high LDL-C levels that had been assessed by a fasting lipid testing during the NHANES.

\section{METHODS}

\section{Study Participants}

NHANES is a continuous survey of the health and nutritional status of the US civilian, noninstitutionalized population; participants are selected through a complex, multistage probability design. ${ }^{4}$ Each year, approximately 6,000 participants are selected to participate in the study. Persons who agree to participate are first interviewed in their homes about their health, disease history, and diet. All interviewed participants are invited to a local Mobile Exam Center for administration of additional questionnaires, physical examinations, and laboratory tests. NHANES data are released in 2-year increments; the present analysis was conducted with data from the 4 most recent study cycles: 1999-2000, 2001-2002, 2003-2004, and 2005-2006. The overall response rates for completed examinations among all screened participants for the study cycles were $76 \%(9,282$ of 12,160$), 80 \%(10,477$ of 13,156$)$, $76 \%(9,643$ of 12,761$)$, and $77 \%(9,950$ of 12,862$)$, respectively. In this study, to increase the sample size and analytic options, data from the 4 study cycles were combined into 1 data set.

Among 39,352 participants invited to the Mobile Exam Center, 13,875 were randomly selected to fast 8 or more hours (up to 24 hours) for laboratory testing. As did the other subsamples, the fasting subsample has its own designated weight, which takes into account the complex survey design, survey nonresponse, and poststratification in representing the US civilian, noninstitutionalized census population. After exclusion of 10,663 participants aged younger than 20 years, men 35 years or older, or women 45 years or older, our study sample consisted of 3,212 participants. Women who had a positive urine pregnancy test or who reported that they were pregnant $(n=462)$, as well as participants with missing lipid profile data or blood pressure data $(n=163)$, were excluded, leaving an analytical sample of 2,587 participants.

\section{Assessment of CHD Risk Factors}

Participants with a self-reported history of CHD (angina or myocardial infarction) were identified as participants with CHD. Those participants with selfreported stroke or diabetes and those with fasting blood glucose levels of $126 \mathrm{mg} / \mathrm{dL}$ or higher were identified as participants with CHD equivalents.

We examined the following 4 CHD risk factors that according to the USPSTF guidelines should be used to determine the eligibility for cholesterol screening in young adults: (1) cigarette smoking (self-reported smoking every day or some days); (2) hypertension (the average of 3 blood pressure measurements from the NHANES physical examination at or exceeding 140/90 mm Hg, or self-reported current use of antihypertensive medication); (3) family history of premature CHD (angina or myocardial infarction) in a firstdegree relative younger than 50 years; and (4) obesity (a body mass index of 30 or greater that was calculated as weight in kilograms divided by the square of the height in meters).

\section{Assessment and Definition of Screening Status}

The cholesterol screening rates were estimated based on the self-reported screening that took place before our study. Participants were asked whether they had ever had their blood cholesterol levels checked and how long it had been since their last cholesterol test. Screening was dichotomized as either (1) never screened or screened 5 or more years ago or (2) screened within the last 5 years. 


\section{Lipid Measurements and Definition of High LDL-C Levels}

We categorized LDL-C levels based on the ATP III risk categories and goals for therapeutic lifestyle changes and drug therapy (Table 1), and we defined high LDL-C levels as levels above the goal for each risk category. The group of participants with high LDL-C levels included those who were eligible for therapeutic lifestyle change and drug therapy. Details on the classification of the study participants into the 3 ATP III risk categories (high, intermediate, and low) are published elsewhere. ${ }^{5}$

For all lipid analyses, frozen venous serum samples were shipped on dry ice to the Lipoprotein Analytical Laboratory at the Johns Hopkins University Hospital, Baltimore, Maryland. ${ }^{6}$ Methods for determining total cholesterol, high-density lipoprotein cholesterol (HDL-C), and triglyceride levels for 1999-2006 NHANES surveys have been described elsewhere. ${ }^{5}$ LDL-C values were calculated from measured values of total cholesterol, triglycerides, and HDL-C according to the Friedewald calculation. All lipid measurements were standardized through the Centers for Disease Control and Prevention-National Heart, Lung, and Blood Institute Lipid Standardization Program. ${ }^{7}$

\section{Assessment and Definition of Sociodemographic Characteristics}

Information on race/ethnicity, health insurance coverage, education, income, and health care visits was obtained by using a structured questionnaire. Race/ ethnicity was categorized as non-Hispanic white, nonHispanic black, Mexican American, and others. The others group included multiple race and other Hispanic. Because the sample was designed to provide estimates for non-Hispanic white, non-Hispanic black, and Mexican American populations of the United States, we included the others group in the analysis but did not report estimates for this group because of its small sample size, heterogeneity, and nonrepresentative nature.

Participants were classified as insured if they reported having private insurance, Medicaid, or Civilian Health and Medical Program of the Uniformed Services (CHAMPUS)/Veterans Affairs insurance. The poverty index ratio (total family income divided by the poverty threshold index adjusted for family size, composition, and location at year of interview) was categorized numerically according to the following descriptions: low (1 or less: family income is less than or equal to the poverty threshold index), medium ( 2 to 3: family income is 2 to 3 times as high as the poverty threshold index); and high (more than 3: family income is more than 3 times as high as the poverty threshold index). Health care access was assessed by responses to the question, "During the past 12 months, how many times have you seen a doctor or other health care professional about your health at a doctor's office, a clinic, hospital emergency room, at home or some other place?" Participants' responses were grouped into 4 categories: $0,1,2$ to 3 , and 4 or more times.

\section{Data Analysis}

Estimated population prevalence and standard errors were calculated using SUDAAN statistical software to account for nonresponse and the complex sampling design. ${ }^{8}$ The significance of a difference in prevalence was assessed by $\chi^{2}$ test. $^{8}$ Adjustment for multiple comparisons was made using the Bonferroni method. ${ }^{9}$ Orthogonal polynomial coefficients that are calculated recursively according to the method of Fisher and Yates were used for testing linear trends. ${ }^{8}$ 
Because the prevalence of screening was less than $50 \%$, Cox regression was used to calculate prevalence proportion ratios and their $95 \%$ confidence intervals (CIs) to examine the association between screening and the predictors. Although Cox regression is used to estimate the cumulative incidence ratio for longitudinal data, it also can be used to estimate the prevalence proportion ratio for cross-sectional data when a risk period is constant (each observation has equal follow-up time) ${ }^{10}$

\section{RESULTS}

Table 2 shows the distribution of sociodemographic characteristics and risk categories in the population of interest. About $82 \%$ of the population had at least a high school education, $85 \%$ had an income above the poverty level, 73\% had medical insurance, and 78\% had accessed health care during the last 12 months. About $60 \%$ of the population had 1 or more CHD risk factors. A higher proportion of women had medical insurance $(79 \%$ vs $65 \%, P<.05)$ and received health care 1 or more times during the last 12 months $(87 \%$ vs $65 \%, P<.05)$ when compared with men. The prevalence of risk factors and NCEP ATP III risk categories was similar among women and men, except for obesity (31\% vs $24 \%$, respectively, $P<.05$ ).

Table 3 displays the rate of cholesterol screening by risk categories. Although the rate of screening for high cholesterol levels among persons without $\mathrm{CHD}$

Table 2. Sociodemographic Characteristics and CHD Risk Factors for Men Aged 20 to 35 Years and Women Aged 20 to 45 Years, NHANES, 1999-2006

\begin{tabular}{|c|c|c|c|c|c|c|c|c|c|}
\hline Characteristic & $\begin{array}{l}\text { Total } \\
\text { No. }\end{array}$ & $\begin{array}{c}\text { All } \\
\% \text { (SE) }\end{array}$ & $\begin{array}{l}\text { Men } \\
\% \text { (SE) }\end{array}$ & $\begin{array}{l}\text { Women } \\
\% \text { (SE) }\end{array}$ & Characteristic & $\begin{array}{l}\text { Total } \\
\text { No. }\end{array}$ & $\begin{array}{c}\text { All } \\
\% \text { (SE) }\end{array}$ & $\begin{array}{l}\text { Men } \\
\% \text { (SE) }\end{array}$ & $\begin{array}{c}\text { Women } \\
\% \text { (SE) }\end{array}$ \\
\hline Sex & & & & & \multicolumn{5}{|c|}{ Times received health care during last $12 \mathrm{mo}^{c}$} \\
\hline Male & 1,041 & $38.8(1.1)$ & 100 & & 0 & 626 & $21.7(0.9)$ & $35(1.5)$ & $13.3(1.0)$ \\
\hline Female & 1,546 & $61.2(1.1)$ & & 100 & 1 & 576 & $22.1(0.8)$ & $25.6(1.3)$ & $20(1.1)$ \\
\hline \multicolumn{5}{|l|}{ Race/ethnicity } & $2-3$ & 1,086 & $44.2(1.1)$ & $32.8(1.5)$ & $51.4(1.3)$ \\
\hline Non-Hispanic & 1,110 & $65.6(1.7)$ & $62.3(2.3)$ & $67.7(1.7)$ & $\geq 4$ & 299 & $12.0(0.7)$ & $6.6(0.9)$ & $15.4(1.0)$ \\
\hline $\begin{array}{l}\text { white } \\
\text { Non-Hispanic } \\
\text { black }\end{array}$ & 587 & $12.6(1.1)$ & $11.5(1.4)$ & $13.3(1.2)$ & $\begin{array}{l}\text { CHD or CHD } \\
\text { equivalent }\end{array}$ & 126 & $4.6(0.4)$ & $2.7(0.6)$ & $5.9(0.7)$ \\
\hline $\begin{array}{l}\text { Mexican- } \\
\text { American }\end{array}$ & 651 & $10.5(1.0)$ & $13.5(1.3)$ & $8.6(0.9)$ & $\begin{array}{l}\text { Risk factors } \\
\text { High blood } \\
\text { pressure }^{\mathrm{e}}\end{array}$ & 286 & $10.9(0.7)$ & $11.2(1.2)$ & $10.6(0.9)$ \\
\hline Other & 239 & $11.3(1.4)$ & $12.7(1.9)$ & $10.4(1.4)$ & Smoking & 557 & $24.1(1.0)$ & $26.9(1.4)$ & $22.3(1.3)$ \\
\hline \multicolumn{5}{|l|}{ Education } & \multirow{2}{*}{$\begin{array}{l}\text { Family history } \\
\text { Obesity }\end{array}$} & 356 & $15.9(0.8)$ & $13.8(1.3)$ & $17.2(1.1)$ \\
\hline $\begin{array}{l}\text { Less than high } \\
\text { school }\end{array}$ & 657 & $18.2(1.1)$ & $20.9(1.7)$ & $16.6(1.3)$ & & 791 & $28.3(1.0)$ & $23.6(1.4)$ & $31.3(1.3)$ \\
\hline High school & 633 & $25.7(1.3)$ & $29.6(2.0)$ & $23.2(1.4)$ & $\geq 2$ & 436 & $17.9(1.1)$ & $17.6(1.4)$ & $18.0(1.3)$ \\
\hline \multirow{2}{*}{$\begin{array}{l}\text { More than high } \\
\text { school }\end{array}$} & \multirow[t]{2}{*}{1,294} & \multirow[t]{2}{*}{$56.0(1.6)$} & \multirow[t]{2}{*}{$49.5(1.8)$} & \multirow[t]{2}{*}{$60.2(2.0)$} & 1 & 965 & $37.3(1.0)$ & $39.6(1.7)$ & $35.9(1.4)$ \\
\hline & & & & & 0 & 1,060 & $40.2(1.1)$ & $40.1(1.8)$ & $40.3(1.3)$ \\
\hline \multicolumn{5}{|l|}{ Poverty index ${ }^{a}$} & \multicolumn{5}{|c|}{ NCEP ATP III risk categories } \\
\hline 1 & 475 & $15.0(0.9)$ & $13.7(1.1)$ & $15.8(1.2)$ & $\mathrm{High}^{\mathrm{h}}$ & 131 & $4.8(0.5)$ & $3.0(0.5)$ & $6.0(0.7)$ \\
\hline $2-3$ & 1,007 & $39.4(1.4)$ & $43.5(2.1)$ & $36.7(1.4)$ & Intermediate $^{i}$ & 300 & $13.1(0.9)$ & $17.7(1.5)$ & $10.1(0.9)$ \\
\hline$\geq 3$ & 921 & $45.7(1.5)$ & $42.8(2)$ & $47.4(1.5)$ & Low $^{j}$ & 2,156 & $82.1(1.1)$ & $79.3(1.6)$ & $83.9(1.1)$ \\
\hline \multicolumn{5}{|l|}{ Medical insurance ${ }^{b}$} & \multirow{3}{*}{$\begin{array}{l}\text { Currently taking } \\
\text { lipid-lowering } \\
\text { medications }\end{array}$} & \multirow[t]{3}{*}{36} & \multirow[t]{3}{*}{$1.7(0.3)$} & \multirow[t]{3}{*}{$N A^{k}$} & \multirow[t]{3}{*}{$2.3(0.5)$} \\
\hline Yes & 1,746 & $73.1(1.2)$ & $64.8(1.8)$ & $78.4(1.3)$ & & & & & \\
\hline No & 841 & $26.9(1.2)$ & $35.2(1.8)$ & $21.6(1.3)$ & & & & & \\
\hline
\end{tabular}

CHAMPUS = Civilian Health and Medical Program of the Uniformed Services; CHD = coronary heart disease; NA = not available; NCEP ATP III = National Cholesterol Education Program Adult Treatment Panel III; NHANES = National Health and Nutrition Examination Survey; SE = standard error.

a Calculated as total family income/poverty threshold index adjusted for family size, composition, and location at year of interview: low ( $\leq 1$ : family income less than or equal to poverty threshold index); medium (2-3: family income 2 to 3 times as high as poverty threshold index); and high ( $>3$ : family income $>3$ times as high as poverty threshold index).

${ }^{\mathrm{b}}$ Having private insurance, Medicaid, or CHAMPUS/Veterans Affairs insurance.

'Assessed by responses to the question, "During the past 12 months, how many times have you seen a doctor or other health care professional about your health at a doctor's office, a clinic, hospital emergency room, at home or some other place?"

d Self-reported coronary heart disease, angina, myocardial infarction, stroke, or diabetes (self-reported or fasting blood glucose $\geq 126 \mathrm{mg} / \mathrm{dL}$ ).

e Systolic blood pressure $>140 \mathrm{~mm} \mathrm{Hg}$, diastolic blood pressure $>90 \mathrm{~mm} \mathrm{Hg}$, or reporting a prescription medication for hypertension.

f Self-reported.

${ }^{g}$ Body mass index $\geq 30 \mathrm{~kg} / \mathrm{m}^{2}$ (weight in kilograms divided by the square of height in meters).

CHD or CHD equivalent or $\geq 2$ major CHD risk factors and a 10-year Framingham risk $>20 \%$.

i Two or more major CHD risk factors and a 10 -year Framingham risk $\leq 20 \%$.

One or no major CHD risk factor.

${ }^{k}$ Relative SE $\geq 30 \%$, estimate is unreliable. 
or a CHD equivalent was higher among women than among men (49\%-53\% vs 30\%-38\%, respectively), no significant difference in screening for cholesterol was observed among persons with 1 or 2 or more risk factors for CHD compared with persons with no risk factors in both sexes. The rate of screening for high cholesterol levels among persons with CHD or a CHD equivalent was less than $70 \%$ but was significantly higher than the screening rate for persons with no risk factors. Adjustment for sociodemographic characteristics in multivariate Cox regression models had only a small effect on screening rates by number of CHD risk factors regardless of sex.

Table 4 shows the prevalence of high LDL-C levels. Prevalence increased with the number of the CHD risk factors; $6.7 \%$ of persons with no risk factors had high LDL-C levels compared with $25.9 \%$ of those with 2 or more risk factors. The highest prevalence of high LDL-C levels (65.1\%) was found among persons with CHD or a CHD equivalent. The prevalence of high LDL-C levels among young adults without CHD risk factors was $10.1 \%$ and $4.6 \%$ for men and women, respectively. Similar to the cholesterol screening, adjustment for sociodemographic characteristics in

\begin{tabular}{|c|c|c|}
\hline Risk Factor ${ }^{a}$ & $\begin{array}{l}\text { Screening } \\
\% \text { (SE) }\end{array}$ & $\begin{array}{l}\text { Screening } \\
\text { Risk Ratio }(95 \% \\
\text { Cl) }\end{array}$ \\
\hline \multicolumn{3}{|l|}{ All } \\
\hline CHD or CHD equivalent ${ }^{d}$ & $67.7(5.7)$ & $1.5(1.1-2.2)$ \\
\hline$\geq 2$ & $47.4(3.0)$ & $1.2(1.0-1.4)$ \\
\hline 1 & $45.1(2.3)$ & $1.2(1.0-1.4)$ \\
\hline 0 & $41.8(1.8)$ & Referent \\
\hline \multicolumn{3}{|l|}{ Men 20-35 y } \\
\hline CHD or CHD equivalent ${ }^{d}$ & $63.6(10.9)$ & $2.40(1.40-4.13)$ \\
\hline$\geq 2$ & $37.9(4.8)$ & $1.30(0.87-1.94)$ \\
\hline 1 & $35.9(2.7)$ & $1.36(1.01-1.84)$ \\
\hline 0 & $30.0(2.4)$ & Referent \\
\hline \multicolumn{3}{|l|}{ Women $20-45$ y } \\
\hline CHD or CHD equivalent ${ }^{d}$ & $68.9(6.6)$ & $1.32(0.89-1.96)$ \\
\hline$\geq 2$ & $53.4(3.9)$ & $1.12(0.90-1.39)$ \\
\hline 1 & $51.6(2.8)$ & $1.10(0.90-1.34)$ \\
\hline 0 & $49.3(2.2)$ & Referent \\
\hline \multicolumn{3}{|c|}{$\begin{array}{l}\mathrm{CHD}=\text { coronary heart disease; } \mathrm{Cl}=\text { confidence interval; NHANES = National } \\
\text { Health and Nutrition Examination Survey; } \mathrm{SE}=\text { standard error. }\end{array}$} \\
\hline \\
\hline \multicolumn{3}{|c|}{$\begin{array}{l}\text { 'N = 2,402 due to missing data. Each model was adjusted for race/ethnicity, } \\
\text { education, poverty status, medical insurance status, and health care access } \\
\text { during last } 12 \text { months, and age (continuous). }\end{array}$} \\
\hline \multicolumn{3}{|c|}{$\begin{array}{l}\text { ¿Self-reported coronary heart disease, angina, myocardial infarction, stroke, o } \\
\text { diabetes (self-reported or fasting blood glucose } \geq 126 \mathrm{mg} / \mathrm{dL} \text { ). }\end{array}$} \\
\hline
\end{tabular}

multivariate Cox regression models had only a small effect on prevalence of high LDL-C levels by number of $\mathrm{CHD}$ risk factors regardless of sex.

\section{DISCUSSION}

According to 1999-2006 NHANES data, approximately $65 \%$ of young adults with $\mathrm{CHD}$ or a $\mathrm{CHD}$ equivalent, $26 \%$ of young adults with 2 or more risk factors, $12 \%$ of young adults with 1 risk factor, and $7 \%$ with no CHD risk factors had a high LDL-C level. Screening, however, for high blood cholesterol levels in young adults, except for persons with CHD or a CHD equivalent, was low: about $50 \%$ for women and less than $40 \%$ for men. The age and sex disparities in the use of preventive services have been reported before. For example, the screening rate increases as a function of age at the population level, ${ }^{11}$ and younger men are significantly less likely than younger women to receive certain preventive services. ${ }^{12}$ In our study, no significant difference was found in screening rates

Table 4. Prevalence and Standard Errors of High LDL-C Levels by Number of Risk Factors Among Men Aged 20 to 35 Years and Women Aged 20 to 45 Years ( $N=2,587$ ), NHANES, 1999-2006

\begin{tabular}{|c|c|c|}
\hline Risk Factors ${ }^{a}$ & $\begin{array}{l}\text { High LDL-Cb } \\
\% \text { (SE) }\end{array}$ & $\begin{array}{c}\text { High LDL-Cb,c } \\
\text { Risk Ratio }(95 \% \mathrm{Cl})\end{array}$ \\
\hline \multicolumn{3}{|l|}{ All } \\
\hline CHD or CHD equivalent ${ }^{d}$ & $65.1(4.2)$ & $12.8(8.8 ; 18.5)$ \\
\hline$\geq 2$ & $25.9(2.6)$ & $4.0(2.7 ; 5.9)$ \\
\hline 1 & $12.5(1.3)$ & $1.8(1.3 ; 2.6)$ \\
\hline 0 & $6.7(0.8)^{\mathrm{e}}$ & Referent \\
\hline \multicolumn{3}{|l|}{ Men $20-35$ y } \\
\hline CHD or CHD equivalent ${ }^{d}$ & $55.1(10.1)$ & $5.6(1.6 ; 11.9)$ \\
\hline$\geq 2$ & $27.5(3.8)$ & $2.8(1.5 ; 5.1)$ \\
\hline 1 & $13.9(1.9)$ & $1.2(0.7-2.1)$ \\
\hline 0 & $10.1(1.7)^{\mathrm{e}}$ & Referent \\
\hline \multicolumn{3}{|l|}{ Women $20-45$ y } \\
\hline CHD or CHD equivalent ${ }^{d}$ & $68(4.9)$ & $21.1(13.4 ; 33.3)$ \\
\hline$\geq 2$ & $24.9(3.1)$ & $5.7(3.5 ; 9.2)$ \\
\hline 1 & $11.6(1.8)$ & $2.6(1.5 ; 4.4)$ \\
\hline 0 & $4.6(0.8)^{\mathrm{e}}$ & Referent \\
\hline
\end{tabular}

$\mathrm{CHD}=$ coronary heart disease; $\mathrm{Cl}=$ confidence interval; $\mathrm{LDL}-\mathrm{C}=$ low-density lipoprotein cholesterol; NHANES = National Health and Nutrition Examination Survey.

a Risk factors: high blood pressure, smoking, family history, and obesity. b LDL-C $\geq 100, \geq 130$, and $\geq 160 \mathrm{mg} / \mathrm{dL}$ for high, intermediate, and low NCEP ATP III risk categories, respectively.

' $N=2,402$ due to missing data. Each model was adjusted for race/ethnicity, education, poverty status, medical insurance status, and health care access during last 12 months, and age (continuous).

dSelf-reported coronary heart disease, angina, myocardial infarction, stroke, or diabetes (self-reported or fasting blood glucose $\geq 126 \mathrm{mg} / \mathrm{dL}$ ).

e Linear trend across risk categories assessed by calculating orthogonal polynomial coefficients according to the method of Fisher and Yates; $P$ value for linear trends $<.001$ 
between young adults with no risk factors and their counterparts with 1 or more risk factors even after adjustment for sociodemographic and health care factors. Because the severity of atherosclerosis in young adults increases with the number of risk factors, ${ }_{1}^{13-15}$ the low screening rates, particularly among young persons with 2 or more risk factors, are of concern. A tendency for more ambulatory care visits has been proposed as a factor explaining higher screening rates among young women than among young men. ${ }^{12}$ The efficiency of population-based approaches in the prevention of CHD is not well studied. ${ }^{16}$ The American Heart Association (AHA) supports public screenings that "meet acceptable criteria for recruitment, reliability of measurement of cholesterol levels, appropriate educational information, properly trained staff and referral." The AHA recognized that small-scale screening at work sites, schools, churches, community centers, or neighborhood clinics have potential for identifying highrisk individuals. The AHA states, however, that the integration of community screening programs into the medical care system is important to ensure test results interpretation and follow-up by the physician responsible for the patient's care. ${ }^{17}$ Future studies are needed to identify how cholesterol screening among young adults can be improved in primary care settings.

In our study, about $59 \%$ of young adults as defined by NCEP screening guidelines (men aged 20 to 35 years and women aged 20 to 45 years) had CHD, a CHD equivalent, or 1 or more of the following risk factors: family history of early CHD, smoking, hypertension, or obesity. Our results also support the need to improve assessment and management of cardiovascular disease risk factors among young adults. Because therapeutic lifestyle changes are indicated more often than is drug therapy for young adults, clinical management of risk factors in this group may be challenging. Health care clinicians perceive limited time, lack of skills, inadequate reimbursement, and difficulties in patient adherence to lifestyle changes as barriers to effective counseling. ${ }^{18}$

These concerns notwithstanding, preventive efforts focused on decreasing the number of CHD risk factors may be essential for preventing CHD later in life and reducing the burden of CHD at the national level. Indeed, as has been shown by results from NHANES III among white, non-Hispanic persons aged 35 to 74 years, only a small proportion of CHD events (about $10 \%$ ) occur among persons with isolated risk factors and LDL-C levels at which drug therapy is not indicated. ${ }^{19}$ Thus, the American Medical Association (AMA) promotes counseling on health behavior in clinical practice by advocating full insurance coverage for counseling for smoking and nicotine dependence, alcohol consumption, healthy diet, and physical activity among adults. ${ }^{20}$ Meanwhile, the AMA suggests incorporating a routine that "requires minimal time, reinforces the importance of the key health behaviors, and refers patients to community resources as necessary." ${ }^{10}$ As with clinical counseling, not all community-based lifestyle interventions have been found to be effective in the prevention of CHD, but some interventions show promising results. ${ }^{21}$ For example, in a study by Roux and colleagues, ${ }^{22}$ community-based interventions to promote physical activity appeared to substantially reduce incidence of CHD over a lifetime (140 to 476 cases per 100,000 persons). Comprehensive analysis of the available prevention strategies might serve as a foundation for improving current and future prevention efforts in young adults.

Results reported in this study should be interpreted with the following limitations in mind. First, a misclassification bias is possible in our study. The NHANES question on family history of heart attack or angina asks about first-degree relatives younger than 50 years regardless of sex instead of using 55 years for men and 65 years for women, ages proposed by the NCEP ATP III. Thus, some participants may be inappropriately placed in the low-risk rather than in the intermediate- or high-risk category. Second, the proportion of participants with CHD or a CHD equivalent may be underestimated in our study because of lack of data on peripheral vascular disease, symptomatic carotid artery disease, and abdominal aortic aneurysm. Our study, however, focused on young adults for whom the prevalence of these conditions is known to be low. ${ }^{23}$ Finally, although the collection and examination of laboratory data were standardized, self-reported data from interviews and questionnaires may be subject to misunderstanding and recall bias.

The findings in this study suggest approximately two-thirds of all young adults have 1 or more cardiovascular risk factors. Among young adults, we found that the prevalence of high cholesterol levels increased with the number of CHD risk factors, but the cholesterol screening rate was less than $50 \%$ regardless of risk status. Our results indicate that improvement of risk assessment and management for cardiovascular disease among young adults through evidence-based clinical and public health interventions is warranted. To develop and implement these interventions successfully, a comprehensive analysis of the currently available prevention strategies is needed.

To read or post commentaries in response to this article, see it online at http://www.annfammed.org/cgi/content/full/8/4/327.

Key words: Coronary heart disease; hyperlipidemia; health promotion; mass screening 
Submitted September 25, 2009; submitted, revised, December 11, 2009; accepted December 31, 2009.

Disclaimer. The findings and conclusions in this report are those of the authors and do not necessarily represent the official position of the Centers for Disease Control and Prevention.

\section{References}

1. U.S. Preventive Services Task Force. (USPSTF). Screening for Lipid Disorders in Adults, Summary of Recommendations. June, 2008. http://www.ahrq.gov/clinic/USpstf/uspschol.htm. Accessed Dec 5, 2009.

2. Third Report of the Expert Panel on Detection. Evaluation, and Treatment of the High Blood Cholesterol in Adults (Adult Treatment Panel III): Executive Summary. http://www.nhlbi.nih.gov/guidelines/ cholesterol/atp_iii.htm. Accessed Dec 5, 2009.

3. Centers for Disease Control and Prevention (CDC). Trends in cholesterol screening and awareness of high blood cholesterol-United States, 1991-2003. MMWR Morb Mortal Wkly Rep. 2005;54(35): 865-870.

4. Centers for Disease Control and Prevention (CDC), National Center for Health Statistics (NCHS). National Health and Nutrition Examination Survey Data. US Department of Health and Human Services, Centers for Disease Control and Prevention, Hyattsville, MD.

5. Kuklina EV, Yoon PW, Keenan NL. Trends in high levels of low-density lipoprotein cholesterol in the United States, 1999-2006. JAMA. 2009;302(19):2104-2110

6. Centers for Disease Control and Prevention (CDC), National Center for Health Statistics (NCHS). National Health and Nutrition Examination Survey Laboratory Protocol. US Department of Health and Human Services, Centers for Disease Control and Prevention, Hyattsville, MD; 1999-2006.

7. Cooper GR, Myers GL. Reference system for cholesterol measurements. Scand J Clin Lab Invest. 1990;50(suppl 198):27-31.

8. SUDAAN Example Manual. Research Triangle Park, NC: Research Triangle Institute; 2004

9. Leon AC. Multiplicity-adjusted sample size requirements: a strategy to maintain statistical power with Bonferroni adjustments. J Clin Psychiatry. 2004;65(11):1511-1514

10. Barros AJ, Hirakata VN. Alternatives for logistic regression in crosssectional studies: an empirical comparison of models that directly estimate the prevalence ratio. BMC Med Res Methodol. 2003;3:21.
11. Disparities in screening for and awareness of high blood cholesterol-United States, 1999-2002. MMWR. 2005;54(5):117-119.

12. Viera AJ, Thorpe JM, Garrett JM. Effects of sex, age, and visits on receipt of preventive healthcare services: a secondary analysis of national data. BMC Health Serv Res. 2006;6:15.

13. Berenson GS, Srinivasan SR, Bao W, Newman WP III, Tracy RE, Wattigney WA. Association between multiple cardiovascular risk factors and atherosclerosis in children and young adults. The Bogalusa Heart Study. N Engl J Med. 1998;338(23):1650-1656.

14. Raitakari OT, Juonala $M$, Kähönen $M$, et al. Cardiovascular risk factors in childhood and carotid artery intima-media thickness in adulthood: the Cardiovascular Risk in Young Finns Study. JAMA. 2003:290(17):2277-2283

15. Paul TK, Srinivasan SR, Wei C, et al. Cardiovascular risk profile of asymptomatic healthy young adults with increased femoral artery intima-media thickness: The Bogalusa Heart Study. Am J Med Sci. 2005;330(3):105-110.

16. Harper S. Rose's Strategy of Preventive Medicine. Geoffrey Rose with commentary by Kay-Tee Khaw and Michael Marmot. Int J Epidemiol. 2009;38:1743-1745

17. American Heart Association. Scientific position cholesterol screening, (adults and children). http://www.americanheart.org/presenter. jhtml?identifier=4504. Accessed Dec 5, 2009.

18. Mosca L, Linfante AH, Benjamin EJ, et al. National study of physician awareness and adherence to cardiovascular disease prevention guidelines. Circulation. 2005;111(4):499-510.

19. Vasan RS, Sullivan LM, Wilson PW, et al. Relative importance of borderline and elevated levels of coronary heart disease risk factors. Ann Intern Med. 2005;142(6):393-402.

20. DiClemente CC, Lianov LS, Moeller SM, Williams J, Yoast RA. Healthier life steps: a clinician-patient guide from the AMA. 2008. http://www.medscape.com/viewprogram/17742_index. Accessed Dec 5, 2009.

21. Lauer MS. Primary prevention of atherosclerotic cardiovascular disease: the high public burden of low individual risk. JAMA. 2007;297(12):1376-1378.

22. Roux L, Pratt M, Tengs TO, et al. Cost effectiveness of communitybased physical activity interventions. Am J Prev Med. 2008;35(6): 578-588.

23. Lloyd-Jones D, Adams R, Carnethon M, et al.; American Heart Association Statistics Committee and Stroke Statistics Subcommittee. Heart disease and stroke statistics-2009 update: a report from the American Heart Association Statistics Committee and Stroke Statistics Subcommittee. Circulation. 2009;119(3):480-486. 